\section{Appendix}

List 1

[ ] Minor gastrointestinal upset

[ ] Fine tremor

[ ] Polyuria

[ ] Polydipsia

[ ] Weight gain

[ ] Oedema

[ ] Goitre

[ ] Raised antidiuretic hormone secretion

[ ] Hypothyroidism

[ ] Hypokalaemia

[ ] ECG changes

[ ] Exacerbation of psoriasis

[ ] Kidney changes
List 2

[ ] Blurred vision

[ ]Anorexia

[ ] Vomiting

[ ] Diarrhoea

[ ] Muscle weakness

[ ] Mild drowsiness

[ ] Sluggishness

[ ] Giddiness

[ ] Ataxia

[ ] Coarse tremor

[ ] Lack of coordination

[ ]Dysarthria

Question 1: Maximum time between lithium estimations...

Question 2: Maximum time between thyroid estimations...

Psychiatric Bulletin (1992), 16, 475-477

\title{
'Emergency' referrals to a South London community mental handicap team (CMHT)
}

\section{S. GrAVESTOCK, Lecturer, Section of Psychiatry of Mental Handicap, Guy's Hospital, London SE1 9RT; and J. BicKNeLL, Emeritus Professor of the Psychiatry of Mental Handicap, St George's Hospital Medical School, London SW17 0RE}

As more people with mental handicaps* $(\mathrm{MH})$ live in the community, in line with government community care policies (Department of Health, 1989), referrals to $\mathrm{CMHTs}^{*}$ and other community based services will increase. Such referrals may be routine, urgent or emergency, come from various sources, and concern health and social care needs.

Wandsworth is a large south London borough with a multi-cultural, mobile general population and many deprived inner city areas. The Wandsworth CMHT is a well-established multidisciplinary community based team serving the specialist health care needs and selected specialist social work needs of adults with MH in the district health authority catchment area. The team has input from community mental handicap nurses, psychiatrists, psychologists, specialist social workers, and other therapists.

The team operates an open referral system and holds weekly team meetings, aiming to work primarily with known clients offering planned assessment,

'Use of the terms people with 'mental handicaps' (MH) and 'CMHT' should be considered broadly interchangeable with the recently adopted terms learning difficulties or disabilities and similar other-named community teams serving such people. inputs and regular reassessment of needs using a developmental/crisis prevention model as described by Sines \& Bicknell (1985) and Wallace (1989). Some CMHTs have considered the implications of also providing crisis responses to meet the more urgent needs of clients and carers, but there has been little research of such work.

\section{The study}

The sample was all 'emergency' referrals received over the six months agreed by the team to fit their previously decided empirical definition of an 'emergency'.

"An unforeseen or rapid occurrence demanding rapid action if the wellbeing of the client or caregroup is to be restored to or maintained at the previous level of functioning."

The aims were:

(a) to monitor emergency referrals, team interventions and client descriptive outcomes within two weeks of referral, using a simple self-devised semi-structured questionnaire completed by the managing team member(s) 
(b) to analyse retrospectively client casenotes for relevant socio-demographic and clinical characteristics

(c) to classify broadly (as below) the team consensus opinion on client outcome at two weeks, by one of the authors (SG) consulting team meeting minutes while considering the definition of an emergency:

successful outcome - client wellbeing restored or maintained

supportive outcome-client wellbeing unchanged; team inputs considered supportive to client or carers

unsuccessful outcome-client wellbeing unchanged or worse.

\section{Findings}

There were 40 emergency referrals concerning 33 different clients, with $27(82 \%)$ referred once and 6 $(18 \%)$ more than once. Results are reported on all 33 clients' initial or only emergency referrals. Numbers and (percentages) refer to clients.

\section{Socio-demographic and clinical characteristics}

The median age was 25 years, range 17-65, with 27 $(82 \%)$ aged $<40$. Twenty $(61 \%)$ were female and $13(39 \%)$ male. Eighteen (54\%) were of UK and 15 $(46 \%)$ of non-UK ethnic origins. Thirteen (39\%) resided in the family home, $6(18 \%)$ lived independently, $6(18 \%)$ in private/voluntary placements, 6 $(18 \%)$ in other placements and $2(6 \%)$ were of "no fixed abode". Overall, $7(21 \%)$ resided in various out of borough placements. One was a new referral, 32 $(97 \%)$ rereferrals and $24(73 \%)$ were receiving active team input.

Clinically assessed level of $\mathrm{MH}$ showed $3(9 \%)$ with borderline MH (IQ 70-80); 15 (45\%) mild MH (50-70); 7 (21\%) moderate MH (35-49); 7 (21\%) severe/profound $\mathrm{MH}(<34)$; and one client without MH. Epilepsy was present in $11(33 \%)$ and unstable in $4(12 \%)$. Sixteen $(48 \%)$ had current ICD-9 psychiatric diagnoses recorded: $6(18 \%)$ manic-depressive psychosis (4 manic [296.0], 2 depressive type [296.1]); $4(12 \%)$ personality disorders $(301) ; 2(6 \%)$ atypical childhood psychosis (299.8); 2 (6\%) psychosis (not otherwise specified) (298.9); 1 (3\%) paranoid schizophrenia (295.3); and 1 (3\%) neurotic depression (300.4).

Challenging behaviours (CB) (Emmerson et al, $1988)$ were present in $16(48 \%): 11(33 \%)$ with physical aggression, 8 (24\%) destructiveness, 7 (21\%) severe stubbornness/obsessionality, $3(9 \%)$ selfinjurious behaviours and $1(3 \%)$ other CB. Seven of these 16 also had ICD-9 psychiatric diagnoses (personality disorders, atypical childhood psychosis etc).
Twenty (61\%) were prescribed psychoactive medication(s): 8 (24\%) anticonvulsants; 8 (24\%) major tranquillisers; $3(9 \%)$ lithium; $3(9 \%)$ antidepressants; and $2(6 \%)$ minor tranquillisers. Globally assessed client social situation stability showed $24(73 \%)$ with unstable residential situations; $13(39 \%)$ needed regular respite care while only $4(12 \%)$ received this; $22(67 \%)$ had regular day placements, which were unstable in $10(30 \%)$ while $11(33 \%)$ had no such provision.

\section{Referrers and assessed reasons for referral}

Informal carers referred 8 (24\%); generic social workers $8(24 \%)$; social services residential/daycare staff $6(18 \%)$; generic health service staff $3(9 \%)$ specialist health service staff $3(9 \%)$; employers/ voluntary agencies $3(9 \%)$; and GPs $2(6 \%)$. Almost all were referred by telephone within office hours.

Fifteen (46\%) were referred with CB and unstable social situations (USS); 8 (24\%) with acute psychiatric illness (4 also with USS); 5 (15\%) with USS only; $4(12 \%)$ with unstable epilepsy, CB and/or USS; 1 caregroup (3\%) with acute bereavement issues. USS included threat of or actual exclusion of clients from residential/day placements, clients acutely at risk of physical/sexual abuse, carers becoming acutely ill and not coping etc.

\section{Team interventions and client descriptive outcomes}

Support/advice was given in all cases within three days of referral; $29(88 \%)$ received assessment visits, $21(63 \%)$ within three days; $9(27 \%)$ had their medication changed; $27(82 \%)$ were referred to other agencies (generic social workers, general psychiatrists, GPs etc.), 24 (73\%) within three days; 22 $(67 \%)$ were supported to remain in their residential placement; $11(33 \%)$ were admitted or placed elsewhere (generic psychiatric wards, specialist NHS unit, social services hostels etc.), 7(21\%) within three days.

Team psychiatrists were involved in managing 24 (73\%); nurses $17(51 \%)$; social workers $13(39 \%)$; and other disciplines (usually as client key-worker) 1-3 each.

\section{Outcome}

The client outcome achieved was considered successful for $20(61 \%)$; supportive for $7(21 \%)$; and unsuccessful for $6(18 \%)$.

\section{Comment}

The findings should be considered suggestive rather than authoritative given the short study period, small selected sample, empirical data, lack of comparison data on non-emergency referrals, and the influences 
of subjective and team biases. Some findings are similar to those of Bouras \& Drummond (1989) concerning all referrals to a nearby community psychiatry of mental handicap service. Our clients also tended to be "known", aged $<40$, living in the family home or independently and to have mild mental handicaps.

Without further comparative studies, we cannot explain the relatively high proportion of females $(61 \%)$, those with non-UK ethnic origins $(46 \%)$, unstable social situations $(82 \%)$, out of borough residency $(21 \%)$, challenging behaviour $(48 \%)$ and functional psychotic disorders $(27 \%)$. The causeeffect relationships between the reasons for referral and how such factors interact in the final common pathway leading to emergency referral remain unclear.

The team impression was confirmed in that several emergency referrals occurred despite their developmental/crisis prevention inputs. However, we do not know how many potential emergency referrals were prevented by such inputs to other clients.

The team were accessible and mostly responded promptly, but at the cost of often setting aside non-emergency work with other clients. 'Emergency' interventions were similar to those for nonemergency referrals besides their speed and the higher admission rate. The use of medication seemed appropriate given client psychiatric diagnoses and epilepsy. The prominent roles of team psychiatrists, nurses, social workers and client key workers were demonstrated in managing emergency referrals, participating in joint/multidisciplinary work, referring to and liaising with generic agencies etc. The good short-term outcomes achieved for most clients followed rapid, skilled and intensive interventions. Longer term outcomes may be very different.

Future similar studies should use a range of standardised longer term outcome indicators that are sensitive to team input/process activities, quality and consumer (clients, carers and referrers) satisfaction (Gravestock et al, 1991). This study's empirical methodology suggests ways of conducting clinical audit, peer review, and feedback in multidisciplinary teams.

These findings suggest that plans for comprehensive community based mental handicap services should include crisis prevention and intervention components. Future services and case management models could then respond effectively to the routine and urgent, health and social care needs of clients and their carers. The divisions of future service responsibilities and client needs should not handicap both present and future service responses.

\section{Acknowledgements}

We wish to thank all members of the Wandsworth Community Team for their encouragement and cooperation in this study. We also greatly appreciated the advice of Dr Nick Bouras.

\section{References}

Bouras, N. \& Drummond, C. (1989) Community psychiatric service in mental handicap. Health Trends, $21,72$.

DePartment OF HEAlth (1989) Caring for People. London: HMSO.

Emerson, E., Cummings, R., Barrett, S. el al (1988) Challenging behaviour and community services. Mental Handicap, 16, 16-19.

Gravestock, S., Holt, G. \& Bouras, N. (1991) Outcome indicators in mental handicap. British Journal of Psychiatry, 159, 294-295.

Sines, D. \& BiCKNell, J. (1985) Caring for mentally Handicapped People in the Community, pp.9-37. London: Harper \& Row.

WALlACE, E. (1989) Preview: The way to ensure care needs are met. Social Work Today, 6 July, 18-19. 